\title{
Tamanho de amostra para caracterização de frutos de Carya illinoinensis
}

\author{
Tales Poletto $^{1 *}$, Vinícius Spolaor Fantinel1, Marlove Fátima Brião Muniz¹, Adriana Falcão Dutra ${ }^{1}$
}

RESUMO: Os frutos da nogueira-pecã apresentam variações em seus caracteres morfológicos mesmo sendo oriundos de uma mesma cultivar. Na comercialização de nozes, há classificações de acordo com suas características, que são utilizadas para definição de preços de mercado. O objetivo deste trabalho foi determinar o tamanho de amostra (número de frutos) necessário para a estimação da média de caracteres de frutos de nogueira-pecã, cultivar Barton. Para isso, foram coletados 170 frutos ao acaso de 25 árvores matrizes em um pomar comercial no município de Tucunduva - RS e mensurados os caracteres: massa total do fruto, massa da casca, massa de amêndoa, rendimento de amêndoa, diâmetro longitudinal, diâmetro transversal e número de frutos $/ \mathrm{kg}$. Os frutos foram pesados individualmente e após, as porções de casca e amêndoa foram separadas e pesadas em balança analítica. Os diâmetros dos frutos foram medidos com paquímetro digital. Foi calculado o tamanho de amostra para as semiamplitudes do intervalo de $1 \%, 2 \%, 3 \%, \ldots, 15 \%$ da estimativa da média, com grau de confiança (1- $\alpha$ ) de $95 \%$. São suficientes 23 frutos para a estimação da média de caracteres dos frutos de nogueria-pecã cultivar Barton, com semiamplitude do intervalo de confiança igual a $10 \%$ da estimativa da média.

Palavras-chave: suficiência amostral, planejamento experimental, nogueira-pecã

\section{Sample size for characterization of Carya illinoinensis fruits}

\begin{abstract}
The pecan fruits present variations in their morphological characters even though they originate from the same cultivar. In the marketing of nuts (fruits), there are classifications according to their characteristics, which are used to define market prices. The objective of this work was to determine the sample size (number of fruits) required for the estimation of the mean number of pecan fruits, Barton. Fruits were collected from 25 matrix trees in orchards in the city of Tucunduva - RS. A total of 170 fruits were collected at random and the following characteristics were measured: total fruit mass, bark mass, almond mass, almond yield, longitudinal diameter, transverse diameter and number of fruits $/ \mathrm{kg}$. The fruits were weighed individually and after the bark and almond portions were separated and weighed with analytical balance. The fruit diameters were measured with a digital caliper. The sample size for the half-magnitudes of the range of $1 \%, 2 \%, 3 \%, \ldots, 15 \%$ of the mean estimate, with a confidence level $(1-\alpha)$ of $95 \%$ was calculated. Twentythree fruits are sufficient for the estimation of the mean number of characters of the Barton pecan nut fruits, with a halfrange of the confidence interval equal to $10 \%$ of the average estimate.
\end{abstract}

Keywords: sample sufficiency, experimental planning, pecan

\section{INTRODUÇÃO}

A nogueira-pecã [Carya illinoinensis (Wangenh.) K. Koch] é uma árvore caducifólia da família Junglandaceae, nativa dos Estados Unidos e México, no entanto, já é cultivada em diversos lugares do mundo objetivando a produção de nozes (POLETTO et al., 2016). Seu cultivo é mais expressivo em países como, os Estados Unidos, México, África do Sul, Argentina e Austrália (POLETTO et al., 2016).

No Brasil, é cultivada na Região Sul até o Estado de Minas Gerais, entretanto, os plantios comerciais, com maiores extensões de área plantada, encontramse nas regiões do Vale do Taquari, Rio Pardo e Central do Estado do Rio Grande do Sul (POLETTO et al., 2014; POLETTO et al., 2015). No Brasil o interesse pela produção desta fruta se da principalmente pela excelente renda que proporciona (POLETTO et al., 2015).

Segundo dados do IBGE (2016) o Rio Grande do Sul produziu $2.498 .000 \mathrm{~kg}$ de frutos perfazendo $48 \%$ da produção nacional, sendo o valor da produção estimado em R\$19.451.000. Mesmo com uma volumosa produção, estes valores ainda não suprem a demanda nacional, sendo necessária a importação da maior parte do que é consumido. O potencial de mercado no Brasil para esta cultura é grande, uma vez que a produção nacional ainda não atingiu o volume suficiente para atender ao consumo interno.

Dentre as dezenas de cultivares cultivadas em solo brasileiro, as mais importantes são: Mahan, Moneymaker, Barton, Shawnee, Cape Fear, Cherokee, Chickasaw, Choktaw, Desirable, Melhorada, Imperial, Importada, Shoshone, Stuart e Jackson. As cultivares possuem características peculiares e podem ser discriminadas por características morfológicas, visualizadas, principalmente, nos frutos.

A variabilidade morfológica dos frutos é destacada pelas múltiplas formas, tamanhos e colorações. O formato varia entre as cultivares e, 
dentro delas, existem frutos alongados, arredondados, oblongos e outros. $\mathrm{Na}$ comercialização de nozes (frutos) há classificações de acordo com a massa da amêndoa, o tamanho da noz e as características da casca, que são utilizadas para definição de preços de mercado. Além disso, a regulagem das máquinas, na indústria de processamento, é feita de acordo com as características dos frutos, uma vez que o objetivo é retirar a amêndoa inteira (CARGNELUTTI FILHO et al., 2015). Portanto, é importante caracterizar os frutos de nogueira-pecã, necessitando-se, para isso, de amostra (parte da população) confiável, pois, como afirma Callegari-Jaques (2003), o tempo necessário para avaliar toda a população, as despesas e o número de pessoas envolvidas são elevados, o que torna o estudo restritivo.

A determinação do tamanho de amostra é importante para a obtenção de estimativas confiáveis, de acordo com a precisão desejada (CARGNELUTTI FILHO et al., 2010). O tamanho de amostra depende da variabilidade dos dados, da confiabilidade desejada e do erro de estimação permitido (SPIEGEL et al., 2004). Estudos sobre dimensionamento amostral já foram realizados para diversas culturas como para a avaliação de caracteres de maçã (TOEBE et al., 2014), para caracteres de feijão guandu - Cajanus cajan L. (FACCO et al., 2016), avaliação de caracteres de frutos de abacaxizeiro - Ananas comosus L. (KRAUSE et al., 2013), para tremoço branco Lupinus albus L. (BURIN et al., 2014), para avaliação da massa e diâmetro de frutos de nogueirapecã (CARGNELUTTI FILHO et al., 2015) e para altura e diâmetro de mudas de nogueira-pecã (CARGNELUTTI FILHO et al., 2014). Assim, o objetivo deste trabalho foi determinar o tamanho de amostra necessário para a estimação da média de caracteres de frutos de nogueira-pecã, cultivar Barton.

\section{MATERIAL E MÉTODOS}

A coleta dos frutos foi realizada em um plantio de nogueira-pecã da cultivar Barton, no município de Tucunduva, no Estado do Rio Grande do Sul. O plantio tinha 10 anos de idade e o espaçamento entre as plantas de $8 \mathrm{~m} \times 8 \mathrm{~m}$, sendo que, $20 \%$ das plantas eram da cultivar Shoshone, utilizada para polinização. O solo do local é classificado como Latossolo Vermelho Distrófico. Segundo Moreno (1961) o clima é do tipo subtropical úmido, com temperatura média anual de $17^{\circ} \mathrm{C}$, com frequência média de 5 a 20 geadas anuais. Apresenta precipitação pluvial anual de $1.725 \mathrm{~mm}$ (Cfa de Köppen) e bem distribuída nas estações do ano.
Apesar disso, nos meses de verão, pode ocorrer déficit hídrico em função da alta evapotranspiração.

Os frutos foram coletados em 25 árvores matrizes, sendo realizada nos ramos dos terços superior, inferior e médio de cada árvore, em seguida foram embalados em sacos de papel, identificados e levados para o Laboratório de Fitopatologia da Universidade Federal de Santa Maria, onde foram alocados em bandejas plásticas e mantidos em temperatura ambiente em local protegido por 15 dias para posterior avaliação.

Em seguida foram escolhidos, aleatoriamente, 170 frutos que foram mensurados os diâmetros longitudinal e transversal, por meio de paquímetro digital. Após isso, os frutos foram pesados em balança digital para obtenção da massa total do fruto. Posteriormente, separou-se as porções de casca e amêndoa e pesou-se, separadamente, para quantificar as massas de casca e de amêndoa. O rendimento de amêndoa foi obtido pela divisão da massa da amêndoa pela massa total do fruto. A determinação da quantidade de frutos por quilograma foi obtida de forma direta, dividindo-se um quilograma pela massa total do fruto.Para cada caractere mensurado foi calculado os valores máximo e mínimo, a média, o intervalo, a mediana, a variância, o desvio-padrão, o erro padrão da média e o coeficiente de variação. Com base nos 170 frutos, calculou-se o tamanho de amostra (n) pela equação1.

$$
\eta=\frac{t_{\alpha / 2}^{2} S^{2}}{(E r r o)^{2}} \quad \text { Equação 1) }
$$

em que: $\eta$ é o tamanho de amostra; $t$ é o valor da distribuição $t$ de Student cuja área à direita é igual a $\alpha / 2$, isto é, o valor de $t$, tal que: $\mathrm{P}(\mathrm{t}>\mathrm{t} \alpha / 2)=\alpha / 2$, com (n-1) graus de liberdade, com $\alpha=5 \%$ de probabilidade de erro; $S^{2}$ é a variância amostral; Erro é o erro de estimação da média. O tamanho de $\operatorname{amostra}(\eta)$ foi calculado para as semiamplitudes do intervalo de confiança (erro de estimação) de $1 \%$, $2 \%, 3 \%, . ., 15 \%$ da média $(\mathrm{m})$, com coeficiente de confiança $(1-\alpha)$ de $95 \%$.

\section{RESULTADOS E DISCUSSÃO}

A análise estatística descritiva, obtida a partir dos 170 frutos amostrados, indicou variabilidade entre os frutos de nogueira-pecã da cultivar Barton (Tabela 1). O coeficiente de variação $(\mathrm{CV})$ oscilou entre $5,21 \%$, para o caractere diâmetro transversal do fruto, até $24,32 \%$ para o caractere massa da casca. Quanto maior o CV, maior a variabilidade e, consequentemente, para mesma precisão, maior deve ser o tamanho de amostra. Logo, caracteres com 
maiores CV necessitarão de tamanhos de amostra maiores em relação aos caracteres com menor $\mathrm{CV}$.

Resultados semelhantes foram encontrados por Cargnelutti Filho et al. (2015), quando avaliaram a massa e diâmetros de frutos de cultivares de nogueira-pecã. Os autores observaram que o coeficiente de variação dos dados para o caractere diâmetro transversal foi o menor entre os caracteres avaliados (massa do fruto, massa da casca, massa da amêndoa, diâmetro longitudinal e diâmetro transversal), para todas as cultivares estudadas, enquanto que massa da amêndoa e massa da casca foram os caracteres que apresentaram os menores valores de coeficiente de variação. $O$ menor intervalo observado entre o valor máximo e mínimo foi de 0,92 para o caractere diâmetro transversal dos frutos, que variou de 1,58 a $2,53 \mathrm{~cm}$. $\mathrm{O}$ maior intervalo foi de 112,48 unidades para o caractere quantidade de frutos/kg, que oscilou de 100,72 a
213,2 unidades. Com isso, observou-se que os caracteres com intervalo de dados maiores, resultaram também, em maior variabilidade e, consequentemente, maior tamanho de amostra. Já para caracteres com intervalos de dados menores o tamanho da amostra também foi menor para uma mesma precisão.

A genética de cada planta determina suas características gerais e seu respectivo potencial produtivo, no entanto, fatores externos como nutrição, fitossanidade, clima, entre outros, condicionam seu desenvolvimento. A formação do fruto e, principalmente da amêndoa, são sensíveis a esses fatores, proporcionando variações em suas características de acordo com as condições que as plantas são submetidas.

Tabela 1 - Mínimo, máximo, média, mediana, intervalo, erro padrão, desvio padrão, variância e coeficiente de variação (CV) da massa total de frutos (MF), massa da casca de frutos (MC), massa das amêndoas (MA), rendimento de amêndoa (RA), diâmetro longitudinal (DL), diâmetro transversal (DT) e quantidade de frutos para formar um $\mathrm{Kg}$ (frutos/kg) de 170 frutos de nogueira-pecã cultivar Barton.

\begin{tabular}{|c|c|c|c|c|c|c|c|}
\hline \multirow{2}{*}{ Estatística } & \multicolumn{7}{|c|}{ } \\
\hline & MF (g) & $\mathrm{MC}(\mathrm{g})$ & MA $(\mathrm{g})$ & $\mathrm{RA}(\%)$ & $\mathrm{DL}(\mathrm{cm})$ & DT $(\mathrm{cm})$ & Frutos $/ \mathrm{Kg}$ \\
\hline$n^{o}$ & 170 & 170 & 170 & 170 & 170 & 170 & 170 \\
\hline Mínimo & 4,69 & 1,73 & 2,71 & 36,37 & 3,98 & 1,58 & 100,72 \\
\hline Máximo & 9,93 & 5,76 & 5,70 & 75,11 & 5,40 & 2,53 & 213,20 \\
\hline Média & 7,66 & 3,40 & 4,22 & 55,30 & 4,75 & 2,18 & 132,60 \\
\hline Intervalo & 5,24 & 4,03 & 2,99 & 38,74 & 1,42 & 0,95 & 112,48 \\
\hline Mediana & 7,61 & 3,33 & 4,35 & 57,32 & 4,86 & 2,12 & 131,41 \\
\hline Variância & 0,94 & 0,70 & 0,39 & 53,03 & 0,06 & 0,01 & 325,62 \\
\hline Desvio-padrão & 0,97 & 0,83 & 0,63 & 7,28 & 0,26 & 0,11 & 18,04 \\
\hline Erro-padrão & 0,07 & 0,06 & 0,05 & 0,56 & 0,02 & 0,01 & 1,38 \\
\hline $\mathrm{CV}(\%)$ & 12,70 & 24,32 & 14,95 & 13,16 & 5,47 & 5,21 & 13,60 \\
\hline
\end{tabular}

De maneira geral, os caracteres massa da casca, massa da amêndoa, rendimento de amêndoa e frutos $/ \mathrm{kg}$ apresentaram coeficientes de variação maiores que os demais (Tabela 1). Essa maior variabilidade sugere que para a obtenção da estimativa da média, com determinada precisão, seja necessário um tamanho de amostra maior para estes caracteres.

O tamanho de amostra (número de frutos) para a estimação da média de cada caractere, com semiamplitude do intervalo de confiança igual a $1 \%$ da estimativa da média e grau de confiança de $95 \%$, oscilou entre 2.275 frutos, para o caractere massa da casca (MC) até 107 frutos para o caractere diâmetro transversal do fruto (Tabela 2). Estes tamanhos de amostra fornecem excelente precisão (erro de estimação de 1\%) das estimativas. Entretanto, são inviáveis devido ao elevado tempo e mão-de-obra exigidos para a mensuração desse número de frutos.

Para estimativas com menor precisão, ou seja, para a semiamplitude do intervalo de confiança de $10 \%$, foi observada redução nos tamanhos de amostra para o caractere diâmetro transversal e diâmetro longitudinal dos frutos.

Para os caracteres massa total, massa da casa, massa da amêndoa e rendimento de amêndoa e frutos/kg o tamanho da amostra é de 23 frutos (Tabela 2). 
Tabela 2 - Tamanho de amostra para estimativa da média com semiamplitudes do intervalo de confiança de $95 \%$ iguais a $1 \%, 2 \%$, $3 \%$..., $15 \%$ da média com base em 170 frutos para caracteres de frutos de nogueira-pecã da cultivar Barton.

\begin{tabular}{lllllllllllllllll}
\hline Caractere $^{(1)}$ & $1 \%$ & $2 \%$ & $3 \%$ & $4 \%$ & $5 \%$ & $6 \%$ & $7 \%$ & $8 \%$ & $9 \%$ & $10 \%$ & $11 \%$ & $12 \%$ & $13 \%$ & $14 \%$ & $15 \%$ \\
\hline MF & 623 & 156 & 69 & 39 & 25 & 17 & 13 & 10 & 8 & 6 & 5 & 4 & 4 & 3 & 3 \\
MC & 2275 & 569 & 253 & 142 & 91 & 63 & 46 & 36 & 28 & 23 & 19 & 16 & 13 & 12 & 10 \\
MA & 861 & 215 & 96 & 54 & 34 & 24 & 18 & 13 & 11 & 9 & 7 & 6 & 5 & 4 & 4 \\
RA & 668 & 167 & 74 & 42 & 27 & 19 & 14 & 10 & 8 & 7 & 6 & 5 & 4 & 3 & 3 \\
DL & 117 & 29 & 13 & 7 & 5 & 3 & 2 & 2 & 1 & 1 & 1 & 1 & 1 & 1 & 1 \\
DT & 107 & 27 & 12 & 7 & 4 & 3 & 2 & 2 & 1 & 1 & 1 & 1 & 1 & 1 \\
Frutos/kg & 713 & 178 & 79 & 45 & 29 & 20 & 15 & 11 & 9 & 7 & 6 & 5 & 4 & 4 \\
\hline
\end{tabular}

(1) Massa total de frutos (MF), massa da casca de frutos (MC), massa das amêndoas (MA), rendimento de amêndoa (RA), diâmetro longitudinal (DL), diâmetro transversal (DT) e quantidade de frutos para formar um $\mathrm{kg}$ (Frutos/kg).

Esta variabilidade entre caracteres foi evidenciada por Toebe et al. (2012), verificando que, em pêssego, são necessários 169 frutos para estimar a firmeza de polpa, 34 frutos para estimar a massa, a acidez e o ratio, 11 frutos para estimar a suculência com erro de estimação de 5\% da média. Toebe et al. (2011) determinaram que para a mensuração da firmeza de polpa e da cor de fundo da epiderme do pêssego 'Eragil', 121 frutos são suficientes para a estimação da média com um erro de estimação de $5 \%$ da média em atmosfera controlada e de $10 \%$ em armazenamento refrigerado. Segundo os mesmos autores, em maçã 'Royal Gala', 24 frutos são suficientes para a estimativa da média da firmeza de polpa e da cor de fundo da epiderme, com erro de estimação de 5\% da média. Krause et al. (2013) relataram que para a avaliação da massa, do comprimento e do diâmetro de frutos de abacaxizeiro, cultivar Pérola, em experimentos com adubação com um erro de estimação de $4 \%$ da média, devem ser amostrados, respectivamente, 83; 35 e 10 frutos em cada uma das parcelas experimentais.

Os tamanhos de amostra, com erros de estimação de 1 a $15 \%$ da média (Tabela 2) possibilitam ao usuário dessas informações dimensionar o tamanho de amostra de seu experimento de acordo com os tratamentos e a precisão desejada. $\mathrm{O}$ pesquisador pode optar por dimensionamentos menores, porém terá precisão inferior. Para se obter uma mesma precisão amostral, necessita-se de um menor tamanho de amostra, para os caracteres relacionados ao diâmetro de frutos (DL e DT), enquanto que, para os caracteres relacionados à massa de frutos $(\mathrm{MF}$, MC e MA, RA e Frutos/kg) o tamanho de amostra deve ser maior.

\section{CONCLUSÃO}

O tamanho de amostra para a estimação da média de caracteres dos frutos de nogueira-pecã cultivar Barton, com semiamplitude do intervalo de confiança igual a $10 \%$ da estimativa da média e grau de confiança de $95 \%$ é de 23 frutos.

\section{AGRADECIMENTOS}

Ao Conselho Nacional de Desenvolvimento Científico e Tecnológico (CNPq), pela concessão de bolsa de Produtividade em Pesquisa para Marlove Fátima Brião Muniz.

\section{REFERÊNCIAS}

BURIN, C.; CARGNELUTTI FILHO, A.; TOEBE, M.; ALVES, B. M.; FICK, A. L. Dimensionamento amostral para a estimação da média de caracteres de tremoço branco. Comunicata Scientiae, v. 5, n. 2, p. 205-212, 2014.

CALLEGARI-JAQUES S. M. Bioestatística: princípios e aplicações. Porto Alegre: Artmed. 2003. 256p.

CARGNELUTTI FILHO, A.; LOPES, S. J.; BRUM, B.; REIS DA SILVEIRA, T.; TOEBE, M.; STORCK, L. Tamanho de amostra de caracteres em híbridos de mamoneira. Ciência Rural, v. 40, n. 2, p. 280-287, 2010.

CARGNELUTTI FILHO, A.; POLETTO, T.; MUNIZ, M. F. B.; BAGGIOTTO, C.; POLETTO, I. Dimensionamento amostral para avaliação da massa e diâmetro de frutos de nogueira-pecã. Ciência Rural, v. 45 , n. 5, p. 794-798, 2015. 
CARGNELUTTI FILHO, A.; POLETTO, T.; BRIÃO MUNIZ, M. F.; BAGGIOTTO, C.; POLETTO, I.; FRONZA, D. Dimensionamento amostral para avaliação de altura e diâmetro de mudas de nogueira-pecã. Ciência Rural, v. 44, n. 12, p. 2151-2156, 2014.

FACCO, G.; CARGNELUTTI FILHO, A.; ALVES, B. M.; BURIN, C.; DOS SANTOS, G. O.; KLEINPAUL, J. A.; NEU, I. M. M. Tamanho de amostra para a estimação da média de caracteres produtivos de feijão guandu. Ciência Rural, v. 46, n. 4, p. 619-625, 2016.

IGBE - INSTITUTO BRASILEIRO DE GEOGRAFIA E ESTATÍSTICA. Produção Agrícola Municipal 2015. Rio de Janeiro: IBGE, 2016. Disponível em: <http://www.ibge.gov.br/estadosat/perfil.php?sigla=rs $>$. Acesso em: 17 de agosto de 2017.

KRAUSE, W.; STORCK, L.; LÚCIO, A. D.; NIED, A. H.; GONÇALVES, R. Q. Tamanho ótimo de amostra para avaliação de caracteres de frutos de abacaxizeiro em experimentos com adubação usando parcelas grandes. Revista Brasileira de Fruticultura, Jaboticabal, v. 35, n. 1, p. 183-190, 2013.

MOREnO, J. A. Clima do Rio Grande do Sul, Porto Alegre: Secretaria da Agricultura, 1961. 41 p.

POletTO, T.; MUNIZ, M. F. B.; BAGGIOTTO, C.; CECONI, D. E.; POLETTO, I. Fungos associados às flores e frutos da nogueira-pecã (Carya illinoinensis). Revista de Ciências Ambientais, v. 8, n. 1, p. 05-13, 2014.
K. Koch. Revista Árvore, v. 39, n. 6, p. 1111-1118, 2015.

POLETTO, T.; MUNIZ, M. F. B.; POLETTO, I.; STEFENON, V. M.; MACIEL, C. G.; RABUSQUE, J. E. Superação de dormência e qualidade de mudas de nogueira-pecã em viveiro. Ciência Rural, v. 46, n. 11, p.1980-1985, 2016.

SPIEGEL, M. R.; SCHILLER, J. J.; SRINIVASAN, R. A. Probabilidade e estatística. 2.ed. Porto Alegre: Bookman, 2004. 398p.

TOEBE, M.; BOTH, V.; BRACKMANN, A.; CARGNELUTTI FILHO, A.; THEWES, F. R. Tamanho de amostra para a estimação da média de caracteres de pêssego na colheita e após o armazenamento refrigerado. Ciência Rural, v. 42, n. 2, p. 209-212, 2012.

TOEBE, M.; BOTH, V.; CARGNELUTTI FILHO, A.; BRACKMANN, A.; STORCK, L. Dimensionamento amostral para avaliar firmeza de polpa e cor da epiderme em pêssego e maçã. Revista Ciência Agronômica, v. 42, n. 4, p. 1026-1035, 2011.

TOEBE, M.; BOTH, V.; THEWES, F. R.; CARGNELUTTI FILHO, A.; BRACKMANN, A. Tamanho de amostra para a estimação da média de caracteres de maçã. Ciência Rural, v. 44 n. 5 p. 759-767, 2014.

POLETTO, T.; BRIÃO MUNIZ, M. F.; POLETTO, I.; BAGGIOTTO, C. Métodos de superação de dormência da semente de nogueira-pecã Carya illinoinensis (Wangenh.) 